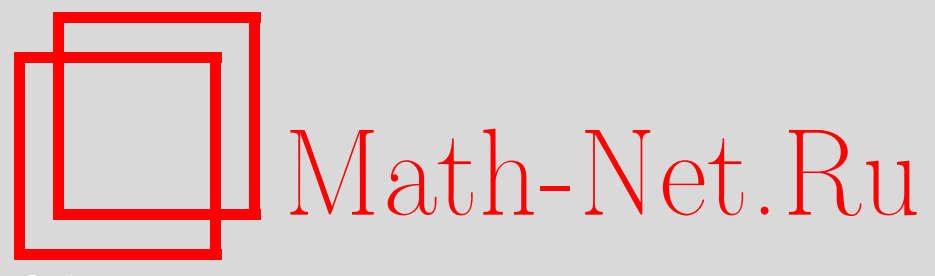

Н. М. Ходжаев, О задаче В.Г. Спринджука, Дискрет. матем., 2003, том 15, выпуск 2, 63-82

DOI: https://doi.org/10.4213/dm194

Использование Общероссийского математического портала Math-Net.Ru подразумевает, что вы прочитали и согласны с пользовательским соглашением http://www.mathnet.ru/rus/agreement

Параметры загрузки:

IP : 54.210 .77 .194

26 апреля 2023 г., 18:03:43

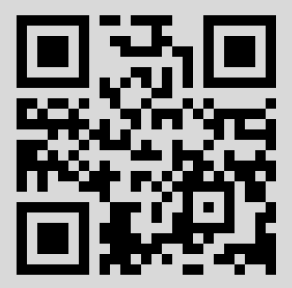


Удк 511.2

\title{
О задаче В. Г. Спринджука
}

\author{
() 2003 г. . Н. М. Ходжаев
}

В статье рассматриваются оценки функции

$$
S(t)=\prod_{p \mid t} p,
$$

бесквадратной части натурального аргумента $t$. В. Г. Спринджуком была поставлена следующая задача: существует ли постоянная $c>0$ такая, что для бесконечно многих пар натуральных чисел $n$ и $k$, удовлетворяющих условию $k<\ln ^{c} n$, справедливо неравенство

$$
S((n+1) \ldots(n+k))<k^{k} ?
$$

В статье доказана следующая теорема. Существуют положительные постоянные $c_{7}, \ldots, c_{10}$ такие, что при $n \geqslant c_{7}$

$$
S((n+1) \ldots(n+k)) \geqslant p_{1} \ldots p_{s(k)}, \quad s(k)=k+\left[c_{8} k / \ln (2 k)\right],
$$

если $1 \leqslant k \leqslant c_{9} \sqrt{n / \ln n}$;

$$
S((n+1) \ldots(n+k))<p_{1} \ldots p_{k},
$$

если $k \geqslant c_{10} \sqrt{n / \ln n}$.

В статье получен ряд других оценок функции $S(t)$, а также рассмотрены некоторые предположения и их следствия, связанные с $S(t)$.

\section{1. Формулировка результатов и предположений}

П. Эрдеш, Т. Н. Шорей и В. Г. Спринджук поставили несколько взаимосвязанных задач о простых делителях биномиальных коэффициентов и произведений последовательных натуральных чисел.

П. Эрдеш [1] предположил, что для натуральных чисел $n$ и $k$

$$
p((n+1) \ldots(n+k))>p_{k+1}
$$

при $n>p_{k+1} \geqslant c_{1}$, где $p(t)$ - наибольший простой делитель целого числа $t$ при $t \geqslant 2$, $p(1)=1, p_{k}-k$-е последовательное простое число.

Это было доказано в более сильной форме К. Рамачандрой [2] и в дальнейшем усилено и обобщено в [3].

Задача Т. Н. Шорея [4] (см. также [5] и [6]) формулируется следующим образом. Для каких натуральных чисел $n$ и $k$ справедливо неравенство

$$
\omega((n+1) \ldots(n+k)) \geqslant k
$$


или более сильное неравенство

$$
\omega((n+1) \ldots(n+k)) \geqslant k+\pi(k)-1,
$$

где $\omega(t)$ - число различных простых делителей целого числа $t, \pi(k)$ - количество простых чисел, не превосходящих $k$ ? К. Рамачандра, Т. Н. Шорей и Р. Тайдеман [5] доказали, что неравенство (2) выполняется при $1 \leqslant k \leqslant e^{c_{2} \sqrt{\ln n}}$, а Ж. Терк (см. [6]) уточнил, что для таких $n$ и $k$

$$
\omega((n+1) \ldots(n+k)) \geqslant k+\pi(k)\left(1-c_{3} \frac{\ln \ln \Delta}{\ln \Delta}\right)
$$

где $\Delta=\ln n / \ln ^{2} k$. П. Эрдеш и Ж. Терк [6] предположили, что неравенство (3) справедливо при $1 \leqslant k \leqslant c_{4} n^{1 / e}$ и оно заменяется на противоположное неравенству (2) при $k \geqslant c_{5} n^{1 / e}$. Они доказали, что это так для бесконечно многих пар натуральных чисел $n$ и $k$ вида $n \asymp N$ с произвольными $k=k(N), N \geqslant c_{6}$.

И, наконец, задача В. Г. Спринджука (см. [7], стр. 240), поставленная им в связи с приведенным выше результатом из [5], формулируется следующим образом. Существует ли постоянная $c>0$ такая, что для бесконечно многих пар натуральных чисел $n$ и $k$, удовлетворяющих условию $k<\ln ^{c} n$, справедливо неравенство

$$
S((n+1) \ldots(n+k))<k^{k},
$$

где

$$
S(t)=\prod_{p \mid t} p
$$

есть бесквадратная часть целого числа $t$ ?

Из приведенного выше результата из [5] и неравенства $S(t) \geqslant p_{1} \ldots p_{\omega(t)}$, справедливого для любого натурального числа $t \geqslant 2$, следует отрицательный ответ на так сформулированный вопрос. В [8] доказано, что неравенство (5) выполняется только если $k \gg \sqrt{n}$. В настоящей работе решена следующая модификация задачи В. Г. Спринджука в духе задач П. Эрдеша (1) и Т. Н. Шорея (2)-(3).

Теорема 1. Существуют положительные постоянные $c_{7}, \ldots, c_{10}$ такие, что при $n \geqslant c_{7}$

$$
S((n+1) \ldots(n+k)) \geqslant p_{1} \ldots p_{s(k)}, \quad s(k)=k+\left[c_{8} k / \ln (2 k)\right],
$$

если $1 \leqslant k \leqslant c_{9} \sqrt{n / \ln n}$

$$
S((n+1) \ldots(n+k))<p_{1} \ldots p_{k}
$$

если $k \geqslant c_{10} \sqrt{n / \ln n}$.

Заметим, что из неравенства (4) следует неравенство (6), из которого, в свою очередь, следует неравенство (1).

Следующее обобщение известной $a b c$-гипотезы (см. [9]) позволило бы получить более точные оценки: если

$$
\varphi(x, y)=\sum_{k=0}^{n} c_{k} x^{k} y^{n-k}
$$


- целочисленная форма, $\omega$ - число различных линейных множителей формы $\varphi(x, y), \mathrm{c}$ точностью до числового сомножителя,

$$
H(\varphi)=\max _{0 \leqslant k \leqslant n}\left|c_{k}\right|
$$

- высота, $x$ и $y$ - целые числа, $(x, y)=1, x y \varphi(x, y) \neq 0, H(x, y)=\max (|x|,|y|)$, то

$$
S(\varphi(x, y)) \gg \frac{H(x, y)^{\omega-2-\varepsilon}}{H(\varphi)^{c(n)}},
$$

где постоянная в знаке $\gg$ зависит только от $\boldsymbol{n}$ и произвольного $\varepsilon>0$.

Заметим, что $a b c$-гипотеза соответствует случаю $\varphi(x, y)=x y(x+y)$, причем с помощью замены переменных из нее легко вывести оценку (7) для кубических форм, полностью разложимых над полем рациональных чисел.

Тривиальная оценка $S(t) \leqslant|t|$ показывает, что оценка (7) является уточнением теоремы Туэ-Зигеля-Рота в одной из ее формулировок. На самом деле оценка (7) равносильна следующему усилению теоремы Малера.

Теорема 2. Предположение (7) равносильно оченке

$$
\prod_{v \in S} \min _{1 \leqslant j \leqslant n}\left|\alpha_{i}-a / q\right|_{v} \gg H^{-c_{1}(n)} \prod_{p \in S} p^{-1-\varepsilon} H(a, q)^{-2-\varepsilon},
$$

где $S$ - произвольное конечное множество нормирований поля рачиональных чисел; $\alpha_{1}, \ldots, \alpha_{n}$ - различные алгебрачческие числа вместе со всеми своими сопряженными числами, а и $q$ - челые числа, $q \neq 0, \alpha_{j} \neq a / q$,

$$
H=\prod_{j=1}^{n} H\left(\alpha_{j}\right)
$$

$H\left(\alpha_{j}\right)$ - высоты, $j=1, \ldots, n$, постоянная в знаке 》зависит только от $n$ и $\varepsilon$.

Легко проверить, что из предположения (7) следует гипотеза Морделла для гиперэллиптических и некоторых более общих кривых с оценкой высот рациональных точек на них, а также гипотеза А. Шинцеля и Р. Тайдемана [10], что если $f$ - целочисленный многочлен, у которого хотя бы три не кратных корня, то для целых $x \geqslant x_{0}(f)$ найдется простое число, не кратно входящее в $f(x)$, то есть диофантово уравнение $f(x)=y^{2} z^{3}$ имеет только конечное число решений в целых $x, y, z, y z \neq 0$.

В свою очередь, из гипотезы А. Шинцеля и Р. Тайдемана следует, что для достаточно больших $n$ не существует полноквадратных троек вида $n, n+1, n+3$ (полноквадратное число - это число, все простые делители которого входят в него с кратностью, не меньшей 2), это - предположение Р. А. Моллина и П. Г. Уолша (см. [11]), откуда А. Грэнвил [11] элементарно вывел теорему Адлемана и Хиз-Брауна, [11], откуда они элементарно вывели, что первый случай теоремы Ферма верен для бесконечно многих простых степеней.

Из предположения (7) следует также существование асимптотической плотности бесквадратных значений многочленов и усиление теоремы 1. 
Теорема 3. Пусть $f$ - челочисленный многочлен без кратных корней, $x \geqslant 1, S_{f}(x)-$ количество натуральных чисел $n \leqslant x$, для которых $f(n)-$ бесквадратное число, $N(m)$ - число решений сравнения $f(t) \equiv 0(\bmod m)$,

$$
c(f)=\prod_{p}\left(1-\frac{N\left(p^{2}\right)}{p^{2}}\right) \neq 0 .
$$

Если предположение (7) верно, то при $x \rightarrow \infty$

$$
S_{f}(x) \sim c(f) x .
$$

Теорема 4. Если предположение (7) верно, то для натуральных чисел $n, k, m$ и произвольного $\varepsilon>0$

$$
S((n+1) \ldots(n+k)) \geqslant \frac{n^{(1-\varepsilon) k-1}}{k^{c_{2}(\varepsilon) k}}
$$

и, кроме того,

$$
\sum_{n>N}\left(\left[\frac{x+y}{n^{m}}\right]-\left[\frac{x}{n^{m}}\right]\right) \ll x^{\varepsilon}
$$

$n p u 1 \leqslant y \leqslant x, N \geqslant y^{c_{3}(\varepsilon) /(m-1)} x^{\varepsilon}$

На самом деле асимптотически точная оценка для $S((n+1) \ldots(n+k))$ по существу равносильна асимптотике для количества бесквадратных чисел на коротких промежутках. А именно, обозначим через $S_{2}(x)$ количество бесквадратных чисел, не превосходящих $x$. Тогда справедливо следующее утверждение.

Теорема 5. Пусть $n \geqslant k \geqslant 3-$ натуральные числа. Тогда

$$
\begin{aligned}
& \mid \ln S((n+1) \ldots(n+k))-k \ln (n / k) \mid \\
&=L \sum_{d \leqslant \ln k}\left|S_{2}\left(\frac{n+k}{d^{2}}\right)-S_{2}\left(\frac{n}{d^{2}}\right)-\frac{6 k}{\pi^{2} d^{2}}\right|+O\left(\frac{k \ln n \ln \ln n}{\ln k}\right),
\end{aligned}
$$

где $\ln k \leqslant L \leqslant \ln n$.

В частности, для любого фиксированного $\alpha, 0<\alpha<1$, при $N \rightarrow \infty$ асимптотика

$$
S((n+1) \ldots(n+k))=\left(\frac{n}{k}\right)^{(1+o(1)) k}
$$

для всех $n \asymp N, k \asymp N^{\alpha}$ равносильна асимптотике

$$
S_{2}(n+k)-S_{2}(n) \sim \frac{6}{\pi^{2}} k
$$

для таких $n u k$.

Из теоремы 5 и соответствующего результата из [12] для распределения бесквадратных чисел на почти всех коротких промежутках следует, что асимптотика (11) верна для почти всех $n \asymp N$ при $\ln k / \ln \ln N \rightarrow \infty, k \leqslant N^{1-\varepsilon}, N \rightarrow \infty$. 
Обобщая результат А. Шаркоци [13]

$$
S\left(\left(\begin{array}{c}
2 n \\
n
\end{array}\right)\right)=\left(\begin{array}{c}
2 n \\
n
\end{array}\right) e^{-\left(c_{11}+o(1)\right) \sqrt{n}}
$$

можно предположить, что при $n \geqslant 2, n^{\varepsilon} \leqslant k \leqslant n$ справедливы еще более точные оценки

$$
S\left(\left(\begin{array}{c}
n+k \\
k
\end{array}\right)\right)=\left(\begin{array}{c}
n+k \\
k
\end{array}\right) \exp \left\{-\sum_{m \geqslant 2} a_{m}\left(k^{1 / m}+n^{1 / m}-(n+k)^{1 / m}\right)+O\left(k^{1 / 4} n^{\varepsilon}\right)\right\},
$$

$$
\begin{aligned}
S((n+1) \ldots(n+k)) & =[n+1, \ldots, n+k] \exp \left\{-k^{1 / 2}-k^{1 / 3}+O\left(k^{1 / 4} n^{\varepsilon}\right)+O\left(k^{2} / n\right)\right\} \\
& =\left(\frac{n}{k}\right)^{k} \exp \left\{(1+\gamma) k+O\left(k^{1 / 2} n^{\varepsilon}\right)+O\left(k^{2} / n\right)\right\} \\
S(n+1) \ldots S(n+k) & =n^{k} \exp \left\{-c_{12} k+O\left(k^{1 / 2} n^{\varepsilon}\right)+O\left(k^{2} / n\right)\right\},
\end{aligned}
$$

где $[n+1, \ldots, n+k]-$ наименьшее общее кратное чисел $n+1, \ldots, n+k$,

$$
a_{2}=-\frac{1}{2} \zeta\left(\frac{1}{2}\right)
$$

$\zeta(s)$ - дзета-функщия Римана, $\gamma=0,57 \ldots$ - постоянная Эйлера,

$$
c_{12}=\sum_{p} \frac{\ln p}{p(p-1)}-\gamma
$$

причем основной вклад в оценки (12) и (13) вносят простые числа кратности 2 из промежутка $\varepsilon \sqrt{k} \leqslant p \leqslant \varepsilon^{-1} \sqrt{k}$, а в оценку (14) - простые числа кратности $k / p+O(1)$ из промежутка $k^{\varepsilon} \leqslant p \leqslant k^{1-\varepsilon}$.

Что касается функций $\omega(t)$ и $p(t)$, тесно связанных с функцией $S(t)$, то из предположения (7) и тривиальных оценок

$$
\begin{aligned}
S(\varphi(x, y) & \leqslant p(\varphi(x, y))^{\omega(\varphi(x, y))} \\
\omega(\varphi(x, y)) & \left.\leqslant(1+o(1)) \frac{\ln |\varphi(x, y)|}{\ln \ln |\varphi(x, y)|} \quad \text { (при }|\varphi(x, y)| \rightarrow \infty\right), \\
|\varphi(x, y)| & \leqslant(n+1) H(\varphi) H(x, y)
\end{aligned}
$$

следует оценка

$$
p(\varphi(x, y)) \gg(\ln H(x, y))^{1-2 / \omega-\varepsilon} .
$$

Если использовать вместо оценки (15) гипотетическую оценку

$$
\left.\omega(\varphi(x, y)) \leqslant(1+o(1)) \frac{\ln H(x, y)}{\ln \ln H(x, y)} \quad \text { при } H(x, y) \rightarrow \infty\right),
$$

обобщая предположение П. Эрдеша и Ж.-Л. Николя (см. [14])

$$
\left.\omega(x)+\omega(x+1) \leqslant(1+o(1)) \frac{\ln x}{\ln \ln x} \quad \text { (при } x \rightarrow \infty\right),
$$


то получается оценка

$$
p(\varphi(x, y)) \gg(\ln H(x, y))^{\omega-2-\varepsilon}
$$

На самом деле неулучшаемой при $\omega \geqslant 3$ является, по-видимому, оценка

$$
p(\varphi(x, y)) \gg(\ln H(x, y))^{\omega-1-\varepsilon},
$$

а при $\omega=2-$ оценка

$$
p(\varphi(x, y)) \ln p(\psi(x, y)) \gg(\ln H(x, y))^{1-\varepsilon},
$$

где $\psi$ - произвольная целочисленная форма без общих линейных множителей с формой $\varphi$, с точностью до числовых сомножителей.

Заметим, что к оценкам (16)-(18) приводят вероятностные соображения, если экстраполировать асимптотические плотности и приближенную независимость событий $\omega\left(\mu_{j} x+v_{j} y\right) \geqslant \omega_{j}$ или, соответственно, $p\left(\mu_{j} x+\lambda_{j} y\right) \leqslant z_{j}$.

\section{2. Доказательство теоремы 1}

Сначала, в лемме 1 сведем оценку $S((n+1) \ldots(n+k))$ снизу к оценкам сверху количества целых чисел на промежутке от $n$ до $n+k$, содержащих простое число с высокой кратностью, которые получим в лемме 2.

Лемма 1. Пусть $n, k, m$ - натуральные числа,

$$
S=\ln S((n+1) \ldots(n+k)), \quad S_{m}=\sum_{n<p^{m} s \leqslant n+k} \ln p,
$$

где суммирование ведется по натуральным числам s и простым числам $p$. Тогда

$$
\begin{aligned}
\sum_{m \geqslant 1} S_{m} & =k \ln n+O\left(\frac{k^{2}}{n}\right), \\
S_{m} & \leqslant S+k \sum_{p \leqslant k} \frac{\ln p}{p^{m}}, \\
S_{1} & =S+k \sum_{p \leqslant k} \frac{\ln p}{p}+O(k) .
\end{aligned}
$$

Доказательство. Если положить $t=p^{m} s$, то

$$
S_{m}=\sum_{n<t \leqslant n+k} \sum_{p^{m} \mid t} \ln p .
$$

Используя свойство функции Мангольдта, получаем, что

$$
\sum_{m \geqslant 1} S_{m}=\sum_{n<t \leqslant n+k} \ln t=\sum_{n<t \leqslant n+k}\left(\ln n+O\left(\frac{k}{n}\right)\right)=k \ln n+O\left(\frac{k^{2}}{n}\right),
$$


то есть получаем оценку (19).

Далее, все простые числа $p \leqslant k$ делят $(n+1) \ldots(n+k)$. Следовательно,

$$
S=\sum_{p \mid(n+1) \ldots(n+k), p>k} \ln p+\sum_{p \leqslant k} \ln p .
$$

При $p>k$ не более чем одно число из промежутка от $n$ до $n+k$ делится на $p$ и, кроме того, при $m \geqslant 1$ делимость на $p^{m}$ сильнее делимости на $p$. Поэтому

$$
\begin{aligned}
S & =\sum_{n<t \leqslant n+k} \sum_{p \mid t, p>k} \ln p+\sum_{p \leqslant k} \ln p \geqslant \sum_{n<t \leqslant n+k} \sum_{p^{m} \mid t, p>k} \ln p+\sum_{p \leqslant k} \ln p \\
& =S_{m}-\sum_{n<s p^{m} \leqslant n_{k}, p \leqslant k} \ln p+\sum_{p \leqslant k} \ln p .
\end{aligned}
$$

Тривиально оценивая последнюю сумму по $s$, находим, что

$$
S \geqslant S_{m}-\sum_{p \leqslant k}\left(\left[\frac{n+k}{p^{m}}\right]-\left[\frac{n}{p^{m}}\right]\right)+\sum_{p \leqslant k} \ln p \geqslant S_{m}-k \sum_{p \leqslant k} \frac{\ln p}{p^{m}} .
$$

Аналогично доказывается противоположное неравенство для случая $m=1$.

Лемма 2. Пусть $n$ и $k$ - натуральные числа, $e^{c_{2} \sqrt{\ln n}} \leqslant k \leqslant(n \ln n)^{1 / 3}, n \geqslant c_{13}$,

$$
M=\left[\frac{\ln (n \ln n)}{\ln \Lambda}\right], \quad \theta=\left\{\frac{\ln (n \ln n)}{\ln \Lambda}\right\}, \quad x=\frac{\ln ^{2} \Lambda}{\ln (n \ln n)},
$$

где $\Lambda$ - произвольный параметр такой, что $e^{c_{2} \sqrt{\ln n}} \leqslant \Lambda \leqslant(n \ln n)^{1 / 3}$. Тогда

$$
\begin{aligned}
\sum_{m \geqslant M} S_{m} \leqslant k & \sum_{m \geqslant M} \sum_{p} \frac{\ln p}{p^{m}}+\left(1-e^{-x}\right)^{-1} \Lambda \exp \{-(1-\theta) x(1-(1-\theta) \ln \Lambda / \ln (n \ln n)\} \\
& \times\left(1+\frac{1}{M}+O\left(e^{-x / 4} \frac{\ln \Lambda}{\ln n}\right)+O\left(e^{-x / 4} \frac{\ln \ln n}{\ln \Lambda}\right)+O\left(\frac{\ln \ln n}{M \ln n}\right)\right) \\
& +O\left(e^{-(1-\theta) x / 4} \Lambda / \ln ^{2} n\right)+O\left(\Lambda^{6 / 7} \ln n\right) \\
S_{M-1} \leqslant & \frac{M \Lambda}{M-1} \exp \left\{\theta x+O\left(\theta^{2} x \ln \Lambda / \ln n\right)\right\}\left(1+O\left(\frac{\ln \ln n}{M \ln n}\right)\right)+O(k)
\end{aligned}
$$

Если же $(n \ln n)^{1 / 3} \leqslant k \leqslant n$, то

$$
\sum_{m \geqslant 2} S_{m} \ll k
$$

Доказательство. Оценим сначала суммы $S_{m}$, разбив их на две, в соответствии с тем $p \leqslant V_{m}$ или $p>V_{m}$ (обрезающий параметр $V_{m} \geqslant 2$ выберем позднее):

$$
S_{m}=\sum_{p \leqslant V_{m}} \ln p \sum_{n / p^{m}<s \leqslant(n+k) / p^{m}} 1+\sum_{s \leqslant(n+k) / V_{m}^{m}(n / s)^{1 / m}<p \leqslant((n+k) / s)^{1 / m}} \ln p .
$$

Очевидно, что

$$
\left(\frac{n+k}{s}\right)^{1 / m} \leqslant\left(\frac{n}{s}\right)^{1 / m}\left(1+\frac{1}{m n}\right) .
$$


Поэтому

$$
\begin{aligned}
S_{m} \leqslant & \sum_{p \leqslant V_{m}}\left(1+\frac{k}{p^{m}}\right) \ln p+\sum_{s \leqslant(n+k) / V_{m}^{m}} \frac{\ln ((n+k) / s}{m}\left(1+\frac{1}{m} k n^{1 / m-1} s^{-1 / m}\right) \\
= & k \sum_{p \leqslant V_{m}} \frac{\ln p}{p^{m}}+V_{m}+O\left(\frac{V_{m}}{\ln ^{3} V_{m}}\right)+\frac{(n+k)\left(\ln V_{m}^{m}+1\right)}{V_{m}^{m} m} \\
& \quad+O\left(\frac{\ln n}{m}\right)+O\left(k V_{m}^{1-m} \ln n\right) .
\end{aligned}
$$

При выполнении условия

$$
M-1 \leqslant m \leqslant \frac{3 \ln (n \ln n)}{2 \ln \Lambda}-1
$$

выберем

$$
V_{m}=\left(\frac{m}{m+1} n \ln n\right)^{1 /(m+1)} .
$$

Если положить $m=M+\mu$, то

$$
\begin{aligned}
\ln V_{m} & =\frac{\ln (n \ln n)-\ln (1+1 / m)}{\ln (n \ln n) / \ln \Lambda+\mu+1-\theta} \leqslant \frac{\ln \Lambda}{1+(\mu+1-\theta) \ln \Lambda / \ln (n \ln n)} \\
& =\ln \Lambda-(\mu+1-\theta) x\left(1-\frac{(\mu+1-\theta) \ln \Lambda}{\ln (n \ln n)(1+(\mu+1-\theta) \ln \Lambda / \ln (n \ln n))}\right), \\
& \frac{n}{V_{m}^{m}}=\left(1+\frac{1}{m}\right) \frac{V_{m}}{\ln n}, \quad \ln V_{m}=\frac{\ln (n \ln n)-\ln (1+1 / m)}{m+1} \gg \ln \Lambda .
\end{aligned}
$$

Итак,

$$
\begin{aligned}
S_{m} \leqslant & k \sum_{p} \frac{\ln p}{p^{m}}+V_{m}+\left(1+\frac{1}{m}\right) V_{m}\left(\frac{\ln (n \ln n)-\ln (1+1 / m)}{m+1}+\frac{1}{m}\right) \\
& +\frac{k\left(\ln V_{m}^{m}+1\right)}{m V_{m}^{m}}+O\left(\frac{V_{m}}{\ln ^{3} V_{m}}\right)+O\left(\frac{\ln n}{m}\right)+O\left(k V_{m}^{1-m} \ln n\right) \\
= & k \sum_{p} \frac{\ln p}{p^{m}}+\left(1+\frac{1}{m}\right) V_{m}+O\left(\frac{V_{m} \ln \ln n}{m \ln n}\right)+O\left(k(n \ln n)^{2 /(m+1)-1} \ln n\right),
\end{aligned}
$$

откуда следует оценка (23).

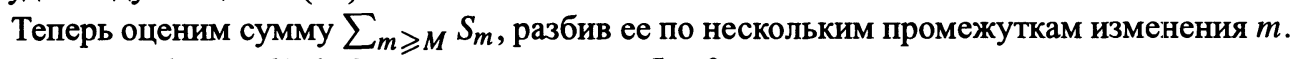

Если $m>\ln (n+k) / \ln 2$, то очевидно, что $S_{m}=0$.

Если

$$
\mu>\frac{\ln (n \ln n)}{2 \ln \Lambda}-1+\theta,
$$

то из определения сумм $S_{m}$ следует, что

$$
p \leqslant(n+k)^{1 / m} \leqslant(n \ln n)^{1 /(3 \ln (n \ln n) /(2 \ln \Lambda)-1)}=\Lambda^{(3 / 2-\ln \Lambda / \ln (n \ln n))^{-1}} \leqslant \Lambda^{6 / 7} .
$$


Поэтому

$$
\begin{aligned}
S_{m} & =\sum_{p} \ln p \sum_{n / \Lambda^{6 / 7}<s \leqslant(n+k) / p^{m}} 1 \leqslant \sum_{p \leqslant \Lambda^{6 / 7}}\left(1+\frac{k}{p^{m}}\right) \ln p \leqslant k \sum_{p} \frac{\ln p}{p^{m}}+O\left(\Lambda^{6 / 7}\right), \\
\sum_{m} S_{m} & \leqslant k \sum_{m} \sum_{p} \frac{\ln p}{p^{m}}+O\left(\Lambda^{6 / 7} \ln n\right),
\end{aligned}
$$

где суммирование по $m$ ведется в интервале

$$
\frac{3 \ln (n \ln n)}{2 \ln \Lambda}-1<m \leqslant \frac{\ln (n+k)}{\ln 2}
$$

В частности, при $\Lambda=(n \ln n)^{1 / 3}$ из оценок (26) и (27) следует оценка (24).

Если

$$
4 \ln \ln n \frac{\ln (n \ln n)}{\ln ^{2} \Lambda}<\mu \leqslant \frac{\ln (n \ln n)}{2 \ln \Lambda}-1+\theta,
$$

то в силу оценки (2)

$$
\ln V_{m} \leqslant \ln \Lambda-(\mu+1-\theta) \frac{x}{2}
$$

Подставляя в оценку (26) и учитывая, что $x \geqslant c_{14}>0$, получаем, что

$$
\begin{aligned}
\sum_{m} S_{m} & \leqslant k \sum_{m} \sum_{p} \frac{\ln p}{p^{m}}+O\left(e^{-(1-\theta) x / 2} \Lambda \sum_{\mu} e^{-\mu x / 2}\right)+O\left(k n^{\varepsilon-1 / 2}\right) \\
& =k \sum_{m} \sum_{p} \frac{\ln p}{p^{m}}+O\left(e^{-(1-\theta) x / 2} \Lambda / \ln ^{2} n\right)+O\left(k n^{\varepsilon-1 / 2}\right),
\end{aligned}
$$

где суммирование ведется по $m$, удовлетворяющим указанным выше неравенствам.

Наконец, если

$$
0 \leqslant \mu \leqslant 4 \ln \ln n \frac{\ln (n \ln n)}{\ln ^{2} \Lambda}
$$

то используем оценку

$$
\begin{aligned}
\ln V_{m} & \leqslant \ln \Lambda-(\mu+1-\theta) x+(\mu+1-\theta)^{2} x \frac{\ln \Lambda}{\ln (n \ln n)} \\
& =\ln \Lambda-(1-\theta) x\left(1-(1-\theta) \frac{\ln \Lambda}{\ln (n \ln n)}\right)-\mu x\left(1-(\mu+2-2 \theta) \frac{\ln \Lambda}{\ln (n \ln n)}\right) \\
& \leqslant \ln \Lambda-(1-\theta) x\left(1-(1-\theta) \frac{\ln \Lambda}{\ln (n \ln n)}\right)-\mu x\left(1-2 \frac{\ln \Lambda}{\ln (n \ln n)}+O\left(\frac{\ln \ln n}{\ln \Lambda}\right)\right) .
\end{aligned}
$$


Подставляя в оценку (26), получаем, что

$$
\begin{aligned}
\sum_{m} S_{m} \leqslant k & \sum_{m} \sum_{p} \frac{\ln p}{p^{m}}+\exp \{-(1-\theta) x(1-(1-\theta) \ln \Lambda / \ln (n \ln n)\} \\
& \times \Lambda \sum_{m} \exp \left\{-\mu x\left(1-2 \frac{\ln \Lambda}{\ln (n \ln n)}+0\left(\frac{\ln \ln n}{\ln \Lambda}\right)\right)\right\} \\
& \times\left(1+\frac{1}{M}+O\left(\frac{\ln \ln n}{M \ln n}\right)\right)+O\left(k n^{\varepsilon-1 / 2}\right) \\
\leqslant & k \sum_{m} \sum_{p} \frac{\ln p}{p^{m}}+\left(1-\exp \left\{-x\left(1-\frac{2 \ln \Lambda}{\ln (n \ln n)}+O\left(\frac{\ln \ln n}{\ln \Lambda}\right)\right)\right\}\right)^{-1} \\
& \times \exp \left\{-(1-\theta) x\left(1-(1-\theta) \frac{\ln \Lambda}{\ln (n \ln n)}\right)\right\} \\
& \times \Lambda\left(1+\frac{1}{M}+O\left(\frac{\ln \ln n}{M \ln n}\right)\right)+O\left(k n^{\varepsilon-1 / 2}\right)
\end{aligned}
$$

причем

$$
\begin{aligned}
\left(1-\exp \left\{-x\left(1-\frac{2 \ln \Lambda}{\ln (n \ln n)}+O\left(\frac{\ln \ln n}{\ln \Lambda}\right)\right)\right\}\right)^{-1} \\
=\left(1-e^{-x}\right)^{-1}\left(1+O\left(e^{-x / 4} \frac{\ln \Lambda}{\ln n}\right)+O\left(e^{-x / 4} \frac{\ln \ln n}{\ln \Lambda}\right)\right) .
\end{aligned}
$$

Собирая оценки (27)-(30), получаем оценку (22).

Лемма 2 доказана.

Доказательство теоремы разобьем на несколько частей по различным промежуткам изменения $k$.

1. Если $1 \leqslant k \leqslant e^{c_{2} \sqrt{\ln n}}$, то оценка (6) теоремы 1 сразу следует из оценки (2).

2. Пусть $e^{c_{2} \sqrt{\ln n}} \leqslant k \leqslant e^{\sqrt{\ln n} \ln \ln n}$. Оценим суммы $S_{m}$ при $1 \leqslant m \leqslant M-1$, используя оценку (20) леммы 1 , а при $m \geqslant M$ используем лемму 2 , полагая $\Lambda=k \ln k$. Тогда

$$
\sum_{m \geqslant 1} S_{m} \leqslant(M-1) S+k \sum_{p \leqslant k} \frac{\ln p}{p-1}+\frac{e^{-(1-\theta) x}}{1-e^{-x}} k \ln k+O\left(k(\ln \ln n)^{4}\right),
$$

причем

$$
\sum_{p \leqslant k} \frac{\ln p}{p-1}=\ln k-\gamma+O(1 / \ln (3 k)) .
$$

Сравнивая полученную оценку с оценкой (19) леммы 1 , получим, что

$$
\begin{aligned}
S \geqslant & \frac{k \ln n-k \ln k-e^{-(1-\theta) x}\left(1-e^{-x}\right)^{-1} k \ln k+O\left(k(\ln \ln n)^{4}\right)}{\ln (n \ln n) / \ln (k \ln k)-1-\theta} \\
= & k \ln (k \ln k)\left(1-(1+\theta) \frac{\ln (k \ln k)}{\ln (n \ln n)}\right)^{-1} \\
& \quad \times\left(1-\frac{\ln (k \ln n)}{\ln (n \ln n)}-\frac{e^{-(1-\theta) x} \ln k}{1-e^{-x} \ln (n \ln n)}+O\left(\frac{(\ln \ln n)^{4}}{\ln n}\right)\right)
\end{aligned}
$$




$$
=k \ln (k \ln k)\left(1+\frac{\ln k}{\ln (n \ln n)}\left(\theta-\frac{e^{-(1-\theta) x}}{1-e^{-x}}\right)+O\left(\frac{(\ln \ln n)^{4}}{\ln n}\right)\right) .
$$

Минимум последнего выражения, как функции от $\theta$, достигается при $\theta=0, \theta=1$ или $\theta=\theta_{0}$, где $e^{\theta_{0} x}=\left(e^{x}-1\right) / x$.

Если $\theta=0$ или $\theta=1$, то

$$
\begin{aligned}
S & \geqslant k \ln (k \ln k)\left(1-\frac{1}{e^{x}-1} \frac{\ln k}{\ln (n \ln n)}+O\left(\frac{(\ln \ln n)^{4}}{\ln n}\right)\right) \\
& =k\left(\ln k+\ln \ln k-\frac{x}{e^{x}-1}+O\left(\frac{\ln k(\ln \ln n)^{4}}{\ln n}\right)\right) .
\end{aligned}
$$

Если же $\theta=\theta_{0}$, то

$$
\begin{aligned}
S & \geqslant k \ln (k \ln k)\left(1+\frac{\ln k}{\ln (n \ln n)}\left(\theta_{0}-\frac{1}{x}\right)+O\left(\frac{(\ln \ln n)^{4}}{\ln n}\right)\right) \\
& =k\left(\ln k+\ln \ln k+x \theta_{0}-1+O\left(\frac{(\ln \ln n)^{4}}{\ln n}\right)\right) .
\end{aligned}
$$

Учитывая, что

$$
x \geqslant c_{15}>0, \quad \frac{x}{e^{x}-1} \leqslant \frac{1}{1+x / 2} \leqslant 1-c_{16}<1, \quad \theta_{0} \geqslant c_{17}>0
$$

и принимая во внимание оценку

$$
p_{s(k)}=k\left(\ln k+\ln \ln k-1+c_{8}+O\left(\frac{\ln \ln k}{\ln k}\right)\right),
$$

которую легко вывести из тождества $s=\pi\left(p_{s}\right)$ и асимптотического ряда для функции $\pi(t)$, получаем утверждение теоремы 1 для значений $k$, рассматриваемых в этом пункте.

3. Пусть $e^{\sqrt{\ln n} \ln \ln n} \leqslant k \leqslant(n \ln n)^{1 / 3}$. Положим $\Lambda=k$ и разобьем доказательство на несколько случаев.

(a) Если $1 / 2 \leqslant \theta<1$, то, как и в случае 2 , из лемм 1 и 2 получаем, что

$$
k \ln n+O\left(k^{2} / n\right)=\sum_{m \geqslant 1} S_{m} \leqslant(M-1) S+k \sum_{p \leqslant k} \frac{\ln p}{p-1}+O(k),
$$

откуда следует, что

$$
\begin{aligned}
S & \geqslant \frac{k \ln n-k \ln k+O(k)}{\ln (n \ln n) / \ln k-1-\theta}=k \ln k\left(1-\frac{3 \ln k}{2 \ln (n \ln n)}\right)^{-1}\left(1-\frac{\ln (k \ln n)+O(1)}{\ln (n \ln n)}\right) \\
& \geqslant k\left(\ln k+\frac{\ln ^{2} k}{2 \ln (n \ln n)}+O\left(\frac{\ln \ln n}{\ln n}\right)\right) .
\end{aligned}
$$

что немного сильнее оценки (6) теоремы 1. 
(b) Если

$$
\frac{\ln \ln n-\ln \ln k+1-\gamma-c / 2}{\ln k}+\frac{\ln \ln k-1+c}{\ln ^{2} k} \ln (n \ln n) \leqslant \theta<\frac{1}{2},
$$

где $c$ - произвольная положительная постоянная, $n>n_{0}(c)$, то снова из лемм 1 и 2 выводим, что

$$
k \ln n+O\left(\frac{k^{2}}{n}\right)=\sum_{m \geqslant 1} S_{m} \leqslant(M-1) S+k \sum_{p \leqslant k} \frac{\ln p}{p-1}+O\left(e^{-x / 4} k\right)+O\left(k^{6 / 7} \ln n\right),
$$

откуда следует, что

$$
\begin{aligned}
S & \geqslant \frac{k \ln n-k \ln k+\gamma k+O(k / \ln k)}{\ln (n \ln n) / \ln k-1-\theta} \\
& =k \ln k \frac{1-(\ln (k \ln n)-\gamma) / \ln (n \ln n)+O(1 /(\ln k \ln n))}{1-(1+\theta) \ln k / \ln (n \ln n)} .
\end{aligned}
$$

Прямая проверка показывает, что

$$
S \geqslant k(\ln k+\ln \ln k-1+c)
$$

(c) Если

$$
\theta \leqslant \frac{\ln \ln n-\ln \ln k+1-\gamma-c / 2}{\ln k}+\frac{\ln \ln k-1+c}{\ln ^{2} k} \ln (n \ln n)
$$

то используя еще оценку (23) леммы 2, получаем, что

$$
\begin{aligned}
k \ln n+O\left(\frac{k^{2}}{n}\right)= & \sum_{m \geqslant 1} S_{m} \leqslant k \sum_{p \leqslant k} \frac{\ln p}{p-1}+(M-2) S \\
& +\frac{M}{M-1} k^{1+\theta \ln k / \ln (n \ln n)+O\left(\theta^{2} \ln ^{2} k / \ln ^{2} n\right)}\left(1+O\left(\frac{\ln \ln n}{M \ln n}\right)\right)+O(k),
\end{aligned}
$$

откуда следует, что

$$
\begin{aligned}
& S \geqslant\left(k \ln n-k \ln k-\frac{M k^{1+\frac{\theta \ln k}{\ln (n \ln n)}+O\left(\frac{\theta^{2} \ln ^{2} k}{\ln ^{2} n}\right)}}{M-1}\left(1+O\left(\frac{\ln \ln n}{M \ln n}\right)\right)+O(k)\right) \\
& \times\left(\frac{\ln (n \ln n)}{\ln k}-2-\theta\right)^{-1} \\
& \geqslant k \ln k\left(1-2 \frac{\ln k}{\ln (n \ln n)}\right)^{-1}\left(1-\frac{\ln (k \ln n)}{\ln (n \ln n)}-\frac{M k^{\frac{\ln \ln k-1+c}{\ln k}}}{(M-1) \ln (n \ln n)}\right. \\
& \left.\times k^{\frac{\ln \ln n-\ln \ln k+1-\gamma-c / 2}{\ln (n \ln n)}+O\left(\left(\frac{\ln \ln k}{\ln k}\right)^{2}\right)}\left(1+O\left(\frac{\ln \ln n}{M \ln n}\right)+O\left(\frac{1}{\ln n}\right)\right)\right)
\end{aligned}
$$




$$
\begin{aligned}
=k & \ln k\left(1-\frac{2 \ln k}{\ln (n \ln n)}\right)^{-1}\left(1-\frac{\ln (k \ln n)}{\ln (n \ln n)}-\frac{M e^{c-1} \ln k}{(M-1) \ln (n \ln n)}\right. \\
& \left.\times\left(e^{1-\gamma-c / 2} \frac{\ln n}{\ln k}\right)^{\ln k / \ln (n \ln n)}\left(1+O\left(\frac{(\ln \ln k)^{2}}{\ln k}\right)+O\left(\frac{1}{\ln n}\right)\right)\right) .
\end{aligned}
$$

В нашем случае

$$
M=\frac{\ln (n \ln n)}{\ln k}-\theta \geqslant(1-\varepsilon) \frac{\ln (n \ln n)}{\ln k} .
$$

Поэтому

$$
S \geqslant k \ln k\left(1-\frac{2(1-\varepsilon)}{M}\right)^{-1}\left(1-\frac{1+\varepsilon}{M}-(1+\varepsilon) \frac{\left(e^{1-\gamma} M\right)^{1 / M}}{M-1} e^{c(1-1 /(2 M))-1}\right) .
$$

Легко проверить, что функция

$$
F(M)=\frac{M}{M-1}\left(e^{1-\gamma} M\right)^{1 / M}
$$

убывает при $M>1$ и $F(3)<e$. Поэтому существует постоянная $c>0$ такая, что при $M \geqslant 3$ выполняется неравенство

$$
S \geqslant k \ln k\left(1+\frac{c_{18}}{M}\right) \geqslant k\left(\ln k+c_{18} \frac{\ln ^{2} k}{\ln (n \ln n)}\right)
$$

более сильное, чем неравенство (6) теоремы 1.

4. Пусть $(n \ln n)^{1 / 3} \leqslant k \leqslant n$. Тогда из оценок (19), (21) и (24) выводим, что

$$
S=k \ln \frac{n}{k}+O(k),
$$

откуда легко следует теорема 1 в рассматриваемой области изменения $k$.

5. Если $k \geqslant n$, то

$$
S((n+1) \ldots(n+k))=\prod_{p \mid(n+1) \ldots(n+k)} p \leqslant \prod_{p \leqslant 2 k} p \leqslant e^{c_{19} k} .
$$

В то же время, $p_{1} \ldots p_{k} \geqslant k !$.

\section{3. Доказательство теоремы 2}

Очевидно, что можно ограничиться случаем, когда многочлен $\varphi(x, 1)$ не имеет кратных корней. Пусть $c$ - старший коэффициент многочлена $\varphi(x, 1)$. Тогда для любого нормирования $v$

$$
|\varphi(x, y)|_{v}=|c|_{v}|y|_{v}^{n} \prod_{j=1}^{n}\left|\alpha_{j}-\frac{x}{y}\right|_{v}
$$

Если

$$
\min _{j}\left|\alpha_{j}-x / y\right|_{v}<\frac{1}{2} \min _{i \neq j}\left|\alpha_{i}-\alpha_{j}\right|_{v}
$$


и минимум достигается при $j=j_{v}$, то

$$
\left|\alpha_{j}-\frac{x}{y}\right|_{v}=\left|\alpha_{j}-\alpha_{j_{v}}+\alpha_{j_{v}}-\frac{x}{y}\right|_{v} \asymp\left|\alpha_{j}-\alpha_{j_{v}}\right|_{v}
$$

при $j \neq j_{v}$. Поэтому,

$$
\left|\alpha_{j_{v}}-\frac{x}{y}\right|_{v} \asymp \frac{|\varphi(x, y)|_{v}}{|c|_{v}|y|_{v}^{n} \prod_{j \neq j_{v}}\left|\alpha_{i}-\alpha_{j_{v}}\right|_{v}} \geqslant|\varphi(x, y)|_{v} \prod_{j \neq j_{v}}\left|\alpha_{j}-\alpha_{j_{v}}\right|_{v}^{-1}
$$

Если же

$$
\min _{j}\left|\alpha_{j}-x / y\right|_{v} \geqslant \frac{1}{2} \min _{i \neq j}\left|\alpha_{i}-\alpha_{j}\right|_{v}
$$

To

$$
|\varphi(x, y)|_{v} \gg|c|_{v}|y|_{v}^{n} \min _{i \neq j}\left|\alpha_{i}-\alpha_{j}\right|^{n}
$$

Предположим теперь, что предположение (7) верно. Тогда

$$
\begin{aligned}
\prod_{p \in S_{1}} p|\varphi(x, y)|_{p} & \geqslant \prod_{p \mid \varphi(x, y)} p^{1-\operatorname{ord}_{p} \varphi(x, y)}=\frac{S(\varphi(x, y))}{|\varphi(x, y)|} \\
& \gg \frac{H(x, y)^{n-2-\varepsilon}}{H(\varphi)^{c(n)}|\varphi(x, y)|} \gg H(\varphi)^{-c(n)-1} H(x, y)^{-2-\varepsilon}
\end{aligned}
$$

для любого конечного множества $S_{1}$ неархимедовых нормирований.

Следовательно, если $S_{1}$ - неархимедовы нормирования из $S$, для которых,

$$
\min _{j}\left|\alpha_{j}-x / y\right|_{p}<\frac{1}{2} \min _{i \neq j}\left|\alpha_{i}-\alpha_{j}\right|_{p}
$$

a $S_{2}$ - остальные неархимедовы нормирования из $S$, то

$$
\begin{aligned}
\prod_{v \in S} \min _{j} \mid \alpha_{j}- & \left.\frac{x}{y}\right|_{v} \gg \min \left(1, \min _{i \neq j}\left|\alpha_{i}-\alpha_{j}\right|, \frac{|\varphi(x, y)|}{|c||y|^{n}} \prod_{j \neq j_{\infty}}\left|\alpha_{j}-\alpha_{j_{\infty}}\right|^{-1}\right) \times \\
& \times H(\varphi)^{-c(n)-1} H(x, y)^{-2-\varepsilon} \prod_{p \in S_{1}} \frac{1}{p} \prod_{j \neq j_{p}}\left|\alpha_{j}-\alpha_{j_{p}}\right|^{-1} \prod_{p \in S_{2}} \min _{i \neq j}\left|\alpha_{i}-\alpha_{j}\right|_{p}
\end{aligned}
$$

Далее, для любого конечного множества $S^{\prime}$ нормирований

$$
\begin{gathered}
\prod_{v \in S^{\prime}} \min _{i \neq j}\left|\alpha_{i}-\alpha_{j}\right|_{v} \geqslant \prod_{i \neq j} \prod_{v \in S^{\prime}} \min \left(1,\left|\alpha_{i}-\alpha_{j}\right|_{v}\right) \geqslant \prod_{i \neq j} H\left(\frac{1}{\alpha_{i}-\alpha_{j}}\right)^{-1}, \\
\prod_{v \in S^{\prime}} \prod_{j \neq j_{v}}\left|\alpha_{j}-\alpha_{j_{v}}\right|^{-1} \geqslant \prod_{i \neq j} H\left(\alpha_{i}-\alpha_{j}\right)^{-1}, \quad \prod_{v \in S^{\prime}}|c|_{v}^{ \pm 1} \geqslant \prod_{j} H\left(\alpha_{j}\right)^{-1} .
\end{gathered}
$$

Для любых алгебраических чисел $\alpha$ и $\beta$, как известно,

$$
H\left(\alpha^{-1}\right) \asymp H(\alpha), \quad H(\alpha-\beta) \ll H(\alpha) H(\beta) .
$$


Поэтому

$$
\begin{aligned}
& \prod_{v \in S^{\prime}} \min _{i \neq j}\left|\alpha_{i}-\alpha_{j}\right|_{v} \gg\left(\prod_{i=1}^{k} H\left(\alpha_{i}\right)\right)^{-2 n}, \\
& \prod_{v \in S^{\prime}} \prod_{j \neq j_{v}}\left|\alpha_{j}-\alpha_{j_{v}}\right|^{-1} \gg\left(\prod_{i=1}^{k} H\left(\alpha_{i}\right)\right)^{-2 n} .
\end{aligned}
$$

Оценки (33)-(35) доказывают оценку (8) теоремы 2.

Докажем теперь, что из оценки (8) следует предположение (7).

Пусть сначала $(x, y)=1,(y, \varphi(x, y))=1$. Выберем $S$ как множество всех простых делителей $\varphi(x, y)$, к которому добавим архимедово нормирование. Тогда из оценки (8) с помощью оценок (31), (32), (34), (35), получаем, что

$$
1=\prod_{v \in S}|\varphi(x, y)|_{v} \gg H(\varphi)^{-c_{1}(n)} H(x, y)^{-2-\varepsilon}|y|^{n} S(\varphi(x, y))^{-1-\varepsilon},
$$

что равносильно оценке (7).

Пусть теперь $(x, y)=1,(y, \varphi(x, y))=d$, но $\varphi(1,0) \neq 0$. Тогда $d \mid c$ и можно для $y=d y^{\prime}$ перейти к новой форме $\varphi^{\prime}\left(x, y^{\prime}\right)=\varphi\left(x, y^{\prime} d\right)$, для которой $\left(y^{\prime}, \varphi^{\prime}\left(x, y^{\prime}\right)\right)=1$, причем $H\left(\varphi^{\prime}\right) \ll d^{n} H(\varphi) \ll H(\varphi)^{n+1}$.

В оставшемся случае $\varphi(1,0)=0$ можно сделать унимодулярное преобразование так, чтобы для новой формы $\varphi^{\prime}(1,0) \neq 0, H\left(\varphi^{\prime}\right) \ll H(\varphi)$.

\section{4. Доказательство теоремы 3}

Очевидно, что

$$
\sum_{d^{2} \mid t} \mu(d)= \begin{cases}1, & \text { если } t-\text { бесквадратное число, } \\ 0 & \text { в противном случае. }\end{cases}
$$

Кроме того,

$$
\sum_{n \leqslant x, f(n) \equiv 0} 1=\frac{N(d)}{d} x+O(N(d)), \quad N(d) \ll d^{\varepsilon}
$$

Поэтому

$$
S_{f}(x)=\sum_{n \leqslant x} \sum_{d^{2} \mid f(n)} \mu(d)=\sum_{d \leqslant x^{2 / 3}} \mu(d)\left(\frac{N\left(d^{2}\right)}{d^{2}} x+O\left(d^{\varepsilon}\right)\right)+O\left(\sum_{n \leqslant x} \sum_{d^{2} \mid f(n), d>x^{2 / 3}} 1\right) .
$$

Учитывая, что в последней сумме

$$
S(f(n)) \leqslant \frac{|f(n)|}{d} \leqslant|f(n)| x^{-2 / 3},
$$

находим, что

$$
S_{f}(x)=c(f) x+O\left(x^{2 / 3+\varepsilon}\right)+O\left(x^{\varepsilon} \sum_{n \leqslant x, S(f(n)) \leqslant|f(n)| x^{-2 / 3}} 1\right) .
$$


Оценим теперь последнюю сумму. Пусть

$$
S(f(n)) \leqslant|f(n)| x^{-2 / 3}, \quad S(f(n+k)) \leqslant|f(n+k)| x^{-2 / 3} .
$$

Если $k \geqslant c_{1}(f)$, то $f(n) f(n+k)$ - многочлен от $n$ без кратных корней с высотой, не превосходящей $k^{c_{2}(f)}$. Поэтому из предположения (7) следует оценка

$$
\frac{|f(n) f(n+k)|}{k^{c_{2}(f)} n^{1+\varepsilon}} \ll S(f(n) f(n+k)) \leqslant|f(n) f(n+k)| x^{-4 / 3},
$$

то есть $k \geqslant x^{c_{3}(f)}, c_{3}(f)>0$.

Итак, натуральные числа $n \leqslant x$, для которых $S(f(n)) \leqslant|f(n)| x^{-2 / 3}$ образуют $O\left(x^{1-c_{3}(f)}\right)$ гругп из $O(1)$ чисел в каждой, что и доказывает теорему 3.

\section{5. Доказательство теоремы 4}

Пусть $k_{0}$ - натуральное число, $k \geqslant k_{0} \geqslant 2$,

$$
\left.N_{i}=\left(n+i k_{0}+1\right)\left(n+i k_{0}+2\right) \ldots\left(n+(i+1) k_{0}\right), \quad i=0,1, \ldots,\left[k / k_{0}\right]-1\right) .
$$

Общие простые делители пар $N_{i}, N_{j}$ содержатся среди простых чисел $p \leqslant k$, поэтому

$$
S((n+1) \ldots(n+k)) \geqslant S\left(\prod_{j=0}^{\left[k / k_{0}\right]-1} N_{j}\right) \geqslant \prod_{j=0}^{\left[k / k_{0}\right]-1}\left(S\left(N_{j}\right) \prod_{p \leqslant k, p \mid N_{j}} p^{-1}\right)
$$

Аналогично, отделяя $p \leqslant k_{0}$, получим, что

$$
\begin{aligned}
\prod_{j=0}^{\left[k / k_{0}\right]-1} \prod_{p \leqslant k, p \mid N_{j}} p & \leqslant \prod_{j=0}^{\left[k / k_{0}\right]-1}\left(\prod_{p \leqslant k_{0}} p \prod_{i=1}^{k_{0}} \prod_{k_{0}<p \leqslant k, p \mid n+j k_{0}+i} p\right) \\
& \leqslant\left(\prod_{p \leqslant k} p\right)^{\left[k / k_{0}\right]-1} \prod_{p \leqslant k} p^{a(p)} \leqslant e^{c_{30} k_{0}\left[k / k_{0}\right]} \prod_{p \leqslant k} p^{k / p+1} \leqslant e^{k \ln k+c_{31} k},
\end{aligned}
$$

где

$$
a(p)=\sum_{n<t \leqslant n+k, p \mid t} 1
$$

Из предположения (7) следует, что

$$
S\left(N_{j}\right) \geqslant c_{5}\left(k_{0}, \varepsilon\right) \frac{n^{k_{0}-1-\varepsilon}}{k^{c_{6}\left(k_{0}\right)}} \geqslant \frac{n^{k_{0}-1-\varepsilon}}{k^{c_{7}\left(k_{0}, \varepsilon\right)}}
$$

Итак,

$$
\begin{aligned}
S((n+1) \ldots(n+k)) & \geqslant e^{-k \ln k-c_{31} k}\left(\frac{n^{k_{0}-1-\varepsilon}}{k^{c_{7}\left(k_{0}, \varepsilon\right)}}\right)^{\left[k / k_{0}\right]} \\
& \geqslant \frac{\left.n^{k\left(1-(1+\varepsilon) / k_{0}\right.}\right)-k_{0}}{k^{c_{8}\left(k_{0}, \varepsilon\right) k}}
\end{aligned}
$$


что равносильно оценке (9) при $k \geqslant k_{0}^{2}, k_{0} \geqslant 1 / \varepsilon$. Для ограниченных $k$ оценка (9) следует из оценки (37).

Теперь докажем, что из предположения (7) следует оценка (10).

Пусть для фиксированного натурального числа $r$ найдется $r$ натуральных чисел $n_{1}, \ldots, n_{r}$ из промежутка $n<n_{j} \leqslant n+k$ таких, что $S\left(n_{j}\right) \leqslant S$. Тогда

$$
S\left(n_{1}, \ldots, n_{r}\right) \leqslant S\left(n_{1}\right) \ldots S\left(n_{r}\right) \leqslant S^{r} .
$$

Если $n_{j}=n+v_{j}$, то из предположения (7) для формы

$$
\varphi(x, y)=y \prod_{j=1}^{r}\left(x+v_{j}\right) y
$$

при $x=n, y=1$ следует, что

$$
S\left(n_{1} \ldots n_{r}\right) \gg_{r} \frac{n^{r-1-\varepsilon}}{k^{c(r)}}
$$

Таким образом,

$$
S k^{c_{9}(r)} \gg_{r} n^{1-1 / r-\varepsilon} \text {. }
$$

Далее,

$$
\sum_{d>N}\left(\left[\frac{n+k}{d^{m}}\right]-\left[\frac{n}{d^{m}}\right]\right)=\sum_{s, d, n<d^{m} s \leqslant n+k, d>N} 1 .
$$

Если положить $v=d^{m} s$, то

$$
S(v) \leqslant d s=\frac{v}{d^{m-1}} \leqslant \frac{2 n}{N^{m-1}} .
$$

Из доказанной ранее оценки (38) следует, что если $n k^{c_{10}(\varepsilon)} / N^{m-1} \ll n^{1-\varepsilon}$, то есть, если $N^{m-1} \gg k^{c_{10}(\varepsilon)} n^{\varepsilon}$, то существует только $O(1)$ чисел $v$, удовлетворяющих условию $S(v) \leqslant 2 n / N^{m-1}$. Поэтому

$$
\sum_{d>N}\left(\left[\frac{n+k}{d^{m}}\right]-\left[\frac{n}{d^{m}}\right]\right) \leqslant \sum_{n<v \leqslant n+k, S(v) \leqslant 2 n / N^{m-1}} d(v) \ll n^{\varepsilon},
$$

где $d(v)$ - количество натуральных делителей числа $v$.

\section{6. Доказательство теоремы 5}

Заметим сначала, что

$$
S(n+1) \ldots S(n+k)=\prod_{t, p, n<t \leqslant n+k, p \mid t} p=\prod_{p} p^{[(n+k) / p]-[n / p]}
$$

и поэтому

$$
\begin{aligned}
S(n+1) \ldots S(n+k) & =S((n+1) \ldots(n+k)) \prod_{p \leqslant k} p^{[(n+k) / p]-[n / p]-1} \\
& =e^{k \ln k+O(k)} S((n+1) \ldots(n+k)) .
\end{aligned}
$$


Пусть теперь

$$
\begin{aligned}
P(t) & =\prod_{p \leqslant t} p, & p_{2}(t) & = \begin{cases}1, & \text { если } t-\text { бесквадратное число, } \\
\max _{p^{2} \mid t} p & \text { в противном случае },\end{cases} \\
N_{p} & =\sum_{n<t \leqslant n+k, p_{2}(t)=p} 1, & N(\Lambda) & =\sum_{n<t \leqslant n+k, p_{2}(t)>\Lambda} 1,
\end{aligned}
$$

где $\Lambda \geqslant 2-$ произвольное число.

Из очевидных неравенств $t / P\left(p_{2}(t)\right) \leqslant S(t) \leqslant t / p_{2}(t)$ следуют неравенства

$$
\begin{aligned}
& S(n+1) \ldots S(n+k) \leqslant(n+1) \ldots(n+k) \Lambda^{-N(\Lambda)} \\
& S(n+1) \ldots S(n+k) \geqslant(n+1) \ldots(n+k) \prod_{p \leqslant \Lambda} P(p)^{-N_{p}}(n+k)^{-N(\Lambda)} .
\end{aligned}
$$

Замечая, что $\ln P(p) \asymp p$ и

$$
N_{p}=\sum_{t} 1 \leqslant \frac{k}{p^{2}}+1,
$$

где суммирование проводится по таким $t$, что $n / p^{2}<t \leqslant(n+k) / p^{2}$ и $l^{2}$ не делит $t$ при $l>p$, получаем, что

$$
\begin{aligned}
& S-k \ln \frac{n}{k} \leqslant-N(\Lambda) \ln \Lambda+O(k), \\
& S-k \ln \frac{n}{k} \geqslant-N(\Lambda) \ln n+O(k \ln \Lambda) .
\end{aligned}
$$

Из тождества для производящего ряда Дирихле

$$
\prod_{p \leqslant \lambda}\left(1-p^{-s}\right)^{-1} \prod_{p>\lambda}\left(1+p^{-s}\right)=\prod_{p}\left(1+p^{-s}\right) \prod_{p \leqslant \lambda}\left(1-p^{-2 s}\right)^{-1},
$$

где $\lambda-$ произвольное число такое, что $2 \leqslant \lambda \leqslant \Lambda$, следует тождество

$$
\begin{aligned}
N(\Lambda)= & k-\sum_{n<t \leqslant n+k, p_{2}(t) \leqslant \Lambda} 1 \\
= & k-\sum_{p(d) \leqslant \lambda}\left(S_{2}\left(\frac{n+k}{d^{2}}\right)-S_{2}\left(\frac{n}{d^{2}}\right)\right)-\sum_{\lambda<p \leqslant \Lambda} N_{p} \\
= & k\left(1-\prod_{p>\lambda}\left(1-p^{2}\right)\right) \\
& \quad-\sum_{p(d) \leqslant \lambda}\left(S_{2}\left(\frac{n+k}{d^{2}}\right)-S_{2}\left(\frac{n}{d^{2}}\right)-\frac{6 k}{\pi^{2} d^{2}}\right)-\sum_{\lambda<p \leqslant \Lambda} N_{p} .
\end{aligned}
$$

Кратность, с которой простое число $p$ входит в $d$, очевидно, не превосходит $\ln d / \ln p$, откуда следует неравенство

$$
\sum_{d^{2} \leqslant n+k, p(d) \leqslant \lambda} 1 \leqslant \prod_{p \leqslant \lambda} \frac{\ln n}{\ln p}
$$


и оценка

$$
\begin{aligned}
N(\Lambda)=- & \sum_{d \leqslant \lambda}\left(S_{2}\left(\frac{n+k}{d^{2}}\right)-S_{2}\left(\frac{n}{d^{2}}\right)-\frac{6 k}{\pi^{2} d^{2}}\right) \\
& +O\left((\ln n)^{\pi(\lambda)}\right)+O(k / \lambda)+O(\Lambda) .
\end{aligned}
$$

Заметим, что

$$
S_{2}(n+k)-S_{2}(n) \leqslant \frac{6 k}{\pi^{2}}+O\left(\frac{k}{\ln k}\right)
$$

для любых $n \geqslant 2, k \geqslant 10$. Действительно, при $z=\ln k$

$$
\begin{aligned}
S_{2}(n+k)-S_{2}(n) & \leqslant \sum_{n<t \leqslant n+k, p^{2} \nmid t \text { for } p \leqslant z} 1 \\
& =\sum_{d \mid P(z)} \mu(d) \sum_{n<t \leqslant n+k, d^{2} \mid t} 1 \\
& =\sum_{d \mid P(z)} \mu(d)\left(k / d^{2}+O(1)\right) \\
& =k \prod_{p \leqslant z}\left(1-p^{2}\right)+O\left(2^{\pi(z)}\right) \\
& =\frac{6 k}{\pi^{2}}+O(k / \ln k) .
\end{aligned}
$$

Следовательно,

$$
N(\Lambda)=\sum_{d \leqslant \ln k / \ln \ln n}\left|S_{2}\left(\frac{n+k}{d^{2}}\right)-S_{2}\left(\frac{n}{d^{2}}\right)-\frac{6 k}{\pi^{2} d^{2}}\right|+O(\Lambda)+O\left(\frac{k}{\ln k} \ln \ln n\right)
$$

при

$$
k \geqslant(\ln n)^{c}, \quad \lambda=\ln k / \ln \ln n .
$$

Осталось выбрать $\Lambda=k / \ln k$ в неравенстве (39) и $\Lambda=\ln k$ в неравенстве (40).

При $k \leqslant(\ln n)^{c}$ утверждение теоремы тривиально.

\section{Список литературы}

1. Эрдеш П., Некоторые проблемы теории чисел. В сб. Вычисления в алгебре и теории чисел. Мир, Москва, 1976, 202-212.

2. Ramachandra K., Largest prime factor of the product of $k$ consecutive integers. Tpydbl Mameм. ин-та им. В. А. Стеклова (1973) 132, 77-81.

3. Turk J., Prime divisors of polynomials at consecutive integers. J. reine und angew. Math. (1980), $319,142-152$.

4. Shorey T. N., On linear form in the logarithms of algebraic numbers. Acta Arithmetica (1976) 31, №1, 27-41.

5. Ramachandra K., Shorey T. N., Tijdeman R., On Grimm's problem relating to factorization of a block of consecutive integers, II. J. reine und angew. Math. (1976) 288, 199-201. 
6. Erdös P., Turk J., Products of integers in short intervals. Acta Arithmetica (1984), 44, №2, 147-174.

7. Спринджук В. Г., Классические диофантовы уравнения от двух переменных. Наука, Москва, 1982.

8. Ходжаев Н. М., Задача В. Г. Спринджука о бесквадратной части произведения послгдовательных натуральных чисел. Вестник МГУ (1997), № 1, 3-7.

9. Lang S., Old and new conjectured Diophantine inequalities. Bulletin Amer. Math. Soc. (1990) 23, №1, 35-75.

10. Shinzel A., Tijdeman R., On the equation $y^{m}=P(x)$. Acta Arithmetica (1976) 31, No 2, 199.

11. Granville A., Powerful numbers and Fermat's last theorem. Math. Rept. Acad. Sci. Can. (1986) 8, № 3, 215-218.

12. Hall R. R., Squarefree numbers on short intervals. Mathematika (1982) 29, № 1, 7-17.

13. Sárközy A., On divisors of binomial coefficients, I. J. Number Theory (1985) 20, № 1, 70-80.

14. Erdös P., Nicolas J.-L., Sur la fonction: Nombre de facteurs premiers de N. Enseign. Math., II Sér. (1981) 27, 3-27.

Статья поступила 28.10.2002. 